\title{
Possible effects of Fierz transformations on vacua of some four fermion interaction models
}

\author{
Bang Rong Zhou \\ College of Physical Sciences, University of the Chinese Academy of Sciences, Beijing, China
}

Email address:

zhoubr@ucas.ac.cn (Bang Rong Zhou)

To cite this article:

Bang Rong Zhou. Possible Effects of Fierz Transformations on Vacua of Some Four Fermion Interaction Models. International Journal of High Energy Physics. Vol. 2, No. 1, 2015, pp. 1-12. doi: 10.11648/j.ijhep.20150201.11

\begin{abstract}
A theoretical research on possible effects of the Fierz transformations on the ground states (vacua) of some 2 flavor four fermion (quark) interaction models has been systematically conducted. It has been shown that, based on the known criterions of the interplay between the antiquark-quark and diquark condensates, in 4D spacetime, for the given antiquark-quark channel couplings with chiral symmetry and from the heavy gluon exchange, the effects of the Fierz transformations are not enough to change the feature that the models' vacua would be in the pure antiquark-quark condensate phases. However, for a given pure scalar diquark channel coupling with the strength $H_{S}$, the Fierz transformations will lead to the nontrivial effect that the model's vacuum could be in the expected diquark condensate phase only if $N_{c}<9$ and $H_{S}$ is small, and as the increase of $N_{c}$ and/or $H_{S}$, the vacuum will get first in a coexistence phase with diquark and antiquark-quark condensates then up to a pure antiquark-quark condensate phase until $N_{c}$ goes to infinite. The similar conclusions are also drawn from relevant four fermion interaction models in 2D and 3D spacetime. The general significance of the research is indicated.
\end{abstract}

Keywords: Four Fermion Interactions, Fierz Transformations, Spinor and $U(N)$ Space,

Antiquark-Quark and Diquark Channels, Color Number $N_{c}$

\section{Introduction}

The four fermion interactions are very useful field theory models to describe dynamical spontaneous breaking [1-6] of symmetries and their restoring at high temperature and high density [7-10] as well as the color superconducting phase transitions at low temperature and high density [11-13]. For the involved four fermion interaction models with dynamical symmetry breaking (from now on the fermion will be called quark), the ground states (vacua) could be in the antiquark-quark $(\bar{q}-q)$ condensate phase or in the diquark $(q-q)$ condensate phase or in the coexistence phase of the above two condensates, depending on the interplay between the $\bar{q}-q$ and $q-q$ condensates in the vacua [14-18]. The presupposition of such interplay is the coexistence of the scalar $\bar{q}-q$ and the scalar or pseudoscalar $q-q$ channel couplings. On the other hand, for any given four fermion couplings, the fermion fields entering them can always be rearranged by the Fierz transformations, thus, by the Fierz transformations, a $\bar{q}-q$ channel coupling will be led to some $q-q$ channel coupling, and the opposite case will also occur. This will inevitably lead to the coexistence of the two kinds of couplings in the resulting effective Lagrangian. Thus a natural question would be drawn out: whether the Fierz transformations could change the feature of the vacuum of a given four fermion interaction model? It seems that a systematical research on this topic has not appeared in the known literature.

The possibility that the diquark condensates could emerge from the vacuum has been researched or touched on by some phenomenological models, including the 2 flavor Quantum Chromodynamics (QCD) instanton-induced NJL model with any $N_{c}$ [14], the random matrix model of 2 flavor and $N_{c}$ color QCD [15] and a 2 flavor color superconducting model [16]. The main results show that such possibility has not been removed theoretically.

To examine further this problem, we have made a more general analysis. Under the assumption that some $\bar{q}-q$ and $q-q$ channel couplings coexist, by means of the effective potential method in the mean field approximation, we have researched the interplay between the $\bar{q}-q$ and $q-q$ condensates in the vacuum respectively for $4 \mathrm{D}, 2 \mathrm{D}$ and $3 \mathrm{D}$ 
four fermion interaction models with flavor $N_{f}=2$ and color $N_{c}=3[17]$ and then extend the discussions to the case of any $N_{c}$ [18], some useful criterions by which the $\bar{q}-q$ and/or $q-q$ condensates could emerge from the vacuum are obtained. However, in the above work, the coexistence of some $\bar{q}-q$ and $q-q$ channel couplings is only an assumption, its possible origin was not be carefully considered. Certainly, the Fierz transformations could be one of the origins, and in fact, as a check of the derived criterions, the Fierz transformations were also briefly mentioned in the Conclusions of [17] for some $N_{c}=3$ models, e.g. 4D chiral invariant model and the heavy gluon exchange models, however, these did not constitute a systematical research on possible effects of the Fierz transformations on the vacua.

In this paper, we will do a systematical research on such effects. In the case of $N_{f}=2$ and keeping $N_{c}$ to be arbitrary, when some four fermion interaction couplings are given, we will examine how their Fierz transformations induce the couplings leading to $\bar{q}-q$ and $q-q$ condensates and how this will affect the vacua of the models. The given starting four fermion couplings, which in 4D case are typical and in most cases, possibly relevant to QCD-like theory, include, besides the chiral invariant model and the heavy gluon exchange model, also the diquark channel coupling which has never been considered before. Because the strengths of the given couplings are assumed to be known, by the Fierz transformations, we will be able to fix uniquely the strengths of the $\bar{q}-q$ and $q-q$ channel couplings in the final effective Lagrangian, including their ratios. This makes it become possible, by means of the general criterions derived in [18], to obtain some definite conclusions of that whether the vacua are actually in the $\bar{q}-q$ or $q-q$ condensate phase or in the coexistence phase of the two condensates. The results will show that in 4D spacetime, for given $\bar{q}-q$ channel couplings, the effects of the Fierz transformations are not enough to change the models' feature that the vacua would be in the pure $\bar{q}-q$ condensate phases. The conclusion seems a little trivial, but it will be demonstrated systematically for the first time. Furthermore, for a given $q-q$ channel coupling, more interesting nontrivial effects will emerge from the Fierz transformations. In this case, the model's vacuum could be in the expected $q-q$ condensate phase only if the $q-q$ channel coupling strength and the color number $N_{c}$ are small enough, otherwise, as the $q-q$ channel coupling strength and/or $N_{c}$ increase, the vacuum would be first in a coexistence phase with $q-q$ and $\bar{q}-q$ condensates and finally up to a pure $\bar{q}-q$ condensate phase. Similar conclusions will also be derived from the 2D and 3D models. This shows some spacetime dimensionality independence of the conclusions. It is emphasized that the basic ideas of the above research, including to relate the effects of the Fierz transformations to the vacua of a class of given four fermion interaction models with dynamical symmetry breaking, and working in the case of any $N_{c}$ and in the 4D, 2D and 3D spacetime, are all original and novel, and most of the obtained results appear in the literature for the first time.

In Sect. 2 we will analyze the effects of the Fierz transformations on scalar and pseudoscalar isovector $\bar{q}-q$ channel couplings, the vectorial $\bar{q}-q$ channel couplings from heavy gluon exchange and scalar $q-q$ channel couplings in 4D spacetime and in Sect.3 and 4, the discussions will be extended to the similarities of the above three couplings in 2D and 3D spacetime. Finally, in Sect.5 we come to our conclusions.

A brief introduction of the Fierz transformations and the explicit expressions of the Fierz transformation matrices as well as corresponding converse forms in spinor spaces of $4 \mathrm{D}$, 2D and 3D spacetime and in flavor or color $U(N)$ space will be given in Appendix. For a given $\bar{q}-q$ channel coupling $\mathcal{L}_{\text {int }}$, its $\bar{q} q \rightarrow \bar{q} q$ and $\bar{q} q \rightarrow q q$ channel Fierz rearrangements will be denoted respectively by $\mathcal{L}_{\text {int }}^{e x}$ (exchange terms) and $\mathcal{L}_{\text {int }}^{q q}$; For a given $q-q$ channel coupling $\mathcal{L}_{q q}$, its $q q \rightarrow \bar{q} q$ channel Fierz rearrangements and corresponding exchange terms will be denoted respectively by $\mathcal{L}_{q q}^{\bar{q} q}$ and $\mathcal{L}_{q q}^{\bar{q} q-e x}$. In the following discussions, for a given coupling, we will always first put down directly the induced total effective Lagrangian after the Fierz transformations and then focus on its physical effects.

\section{4D Four Fermion Interactions}

\subsection{Scalar and Pseudoscalar Isovector Antiquark-Quark Channel Couplings}

The corresponding Lagrangian may be expressed by [1]

$$
{ }_{4(S+P \tau)}=G\left[(\bar{q} q)^{2}+\left(\overline{q i} \gamma_{5} \tau_{a} q\right)^{2}\right]
$$

where $\tau_{a}\left(a=1, \cdots, N_{f}-1\right)$ are the generators of the flavor group $S U_{f}\left(N_{f}\right)$. In present paper, the summation of a Lorentz index is implied to combine into a Lorentz scalar and the summation of an index of the $S U(N)$ generator, unless specified otherwise, will always run over from 1 to $N^{2}-1$. When $N_{f}=2$, the above $\mathcal{L}_{4(S+P \tau)}$ is chiral $S U_{f L}(2) \otimes S U_{f R}(2)$ invarant. $^{1}$ By using the transformations

\footnotetext{
${ }^{1}$ This chiral symmetry reproduces the one of QCD with $N_{c} \geq 3$. Hence (1) can be related to QCD with $N_{c} \geq 3$. However, it can not simulate $N_{c}=2$ QCD with massless quarks, because the latter's chiral symmetry is the higher $S U(4) \quad[12$, 19-22]. It is easy to check that $\mathcal{L}_{4(S+P \tau)}$ in (1) does not have the $S U(4)$ symmetry. For instance, it is only a part of the whole instanton-induced four fermion couplings which are $S U(4)$ invariant when $N_{c}=2$ [21]. Based on the same grounds, the conclusions in $N_{c}=2$ case in present section are merely applicable for the given models here and not for $N_{c}=2$ QCD. In fact, the models considered here only the extensions of some $N_{c}=2$ QCD-relevant four
} 
(A.8), (A.17), (A.9) and (A.18) in the Appendix, we can obtain respectively the Fierz rearranged $\mathcal{L}_{4(S+P \tau)}^{e x}$ (exchange terms) and $\mathcal{L}_{4(S+P \tau)}^{q q}$, thus the total effective Lagrangian becomes

$$
\begin{aligned}
& L_{4(S+P \tau)}^{e f f}=L_{4(S+P \tau)}+L_{4(S+P \tau)}^{e x}+L_{4(S+P \tau)}^{q q} \\
& =G_{S}(\bar{q} q)^{2}+G_{P}\left(\bar{q} i \gamma_{5} q\right)^{2}+G_{P \tau}\left(\bar{q} i \gamma_{5} \tau_{a} q\right)^{2} \\
& \quad+H_{S}\left(\bar{q} i \gamma_{5} \tau_{A} \lambda_{A^{\prime}} q^{c}\right)\left(\bar{q}^{c} i \gamma_{5} \tau_{A} \lambda_{A^{\prime}} q\right) \\
& \quad+H_{P}\left(\bar{q} \tau_{A} \lambda_{A^{\prime}} q^{c}\right)\left(\bar{q}^{c} \tau_{A} \lambda_{A^{\prime}} q\right)+\cdots
\end{aligned}
$$

where we only display part of terms which could be physically interesting, $\tau_{A}$ and $\lambda_{A^{\prime}}$ are separately the antisymmetric generators of the groups $S U_{f}\left(N_{f}\right)$ and $S U_{c}\left(N_{c}\right)$ and the ellipsis stands for all the other possible coupling, where $\lambda_{a}\left(a=1, \cdots, N_{c}-1\right)$ are the generators of $S U_{c}\left(N_{c}\right)$. It is emphasized that $\mathcal{L}_{4(S+P \tau)}^{\text {eff }}$ must be used in Hartree approximation. When $N_{f}=2$, the coupling constants in (2) have the following explicit expressions:

$$
\begin{aligned}
& G_{S}=G_{P \tau}=\left(1+1 / 4 N_{c}\right) G, G_{P}=-G / 4 N_{c}, \\
& H_{S}=-H_{P}=G / 4 .
\end{aligned}
$$

Equation (3) shows that, for the two flavor and $N_{c}$ color model, the induced scalar $q-q$ channel interactions have a positive coupling constant $H_{S}$, however, compared with the scalar $\bar{q}-q$ channel interactions with the coupling constant $G_{S}$, we always have the ratio

$$
G_{S} / H_{S}=\left(4 N_{c}+1\right) / N_{c}>2 / N_{c} .
$$

Thus, based on the general criterion of interplay between the $\bar{q}-q$ and $q-q$ condensates [18], it is impossible to exist the scalar diquark condensates in the vacuum of this model. Such conclusion is also valid in the limit of $N_{c}=2$. It is indicated that, when $N_{c}=2$, the scalar $\bar{q}-q$ condensates $\langle\bar{q} q\rangle$ and the scalar $q-q$ condensates $\left\langle\bar{q} i \gamma_{5} \tau_{2} \lambda_{2} q^{c}\right\rangle$ are both $S U_{f}(2) \otimes S U_{c}(2)$ singlets, however , the former breaks $S U_{f L}(2) \otimes S U_{f R}(2) \quad$ chiral symmetry but the latter conserves it. Hence in the case of $N_{c}=2$ we also have spontaneous breaking of the chiral symmetry. In addition, it is noted that, after the Fierz transformations, the largest attractive channel couplings are still the terms $(\bar{q} q)^{2}$ and $\left(\bar{q} i \gamma_{5} \tau_{a} q\right)^{2}$ with the same coupling strength $G_{S}=G_{P \tau}$, this fact certainly keeps the basic feature of the original $\mathcal{L}_{4(S+P \tau)}$ in (1), including its

fermion interaction models to any $N_{c}$ case and are not supposed to touch the very special $N_{c}=2$ QCD theory. chiral symmetry.

\subsection{Four Fermion Interactions from Heavy Gluon Exchange}

The corresponding Lagrangian is assumed to be [13]

$$
\mathcal{L}_{4(V \lambda)}=-g\left(\bar{q} \gamma^{\mu} \lambda_{a} q\right)\left(\bar{q} \gamma_{\mu} \lambda_{a} q\right)
$$

with the constant $g$. It simulates the interactions induced by one gluon exchange in QCD. Similar to the steps taken in section 2.1., we can obtain the total effective Lagrangian for $N_{f}=2$

$$
\begin{aligned}
\mathcal{L}_{4(V \lambda)}^{e f f}= & \mathcal{L}_{4(V \lambda)}+\mathcal{L}_{4(V \lambda)}^{e x}+\mathcal{L}_{4(V \lambda)}^{q q} \\
= & G_{S}(\bar{q} q)^{2}+G_{V \lambda}\left(\bar{q} \gamma^{\mu} \lambda_{a} q\right)^{2} \\
& +H_{S}\left(\bar{q} i \gamma_{5} \tau_{A} \lambda_{A^{\prime}} q^{c}\right)\left(\bar{q}^{c} i \gamma_{5} \tau_{A} \lambda_{A^{\prime}} q\right)+\cdots
\end{aligned}
$$

with

$$
\begin{aligned}
& G_{S}=\left(N_{c}^{2}-1\right) \mathrm{g} / N_{c}^{2}=2\left(N_{c}-1\right) H_{S} / N_{c}, \\
& G_{V \lambda}=-\left(1-1 / 4 N_{c}\right) g .
\end{aligned}
$$

Hence, the Fierz transformations have induced the scalar $\bar{q}-q$ channel coupling and the scalar $q-q$ channel coupling, however, the corresponding coupling constants $G_{S}$ and $H_{S}$, in the case of $N_{f}=2$ and any $N_{c}$, have the ratio

$$
G_{S} / H_{S}=2\left(N_{c}-1\right) / N_{c}>2 / N_{c} \text {, if } N_{c}>2 \text {. }
$$

This result was given in the Appendix A of [13]. Thus, based on the general criterion given in [18], if $N_{c} \geq 3$, the ground state (vacuum) of the model could only be in antiquark-quark condensate phase. In the limit of $N_{c}=2$, $G_{S} / H_{S}=1$. This implies that we will be at a critical point between breaking and restoring of the chiral symmetry. Once there are the other couplings included, such balance would be broken and the system could come to the phase of either chiral symmetry breaking or chiral symmetry restoring, depending on the feature of the included couplings.

\subsection{Scalar Diquark Channel Interactions}

For describing two flavor color superconductors, one introduces the pure scalar $q-q$ channel coupling with the Lag rangian $[13,18]$

$$
\mathcal{L}_{4\left(S_{q q}\right)}=H_{S}\left(\bar{q} i \gamma_{5} \tau_{A} \lambda_{A^{\prime}} q^{c}\right)\left(\bar{q}^{c} i \gamma_{5} \tau_{A} \lambda_{A^{\prime}} q\right) .
$$

Equation (9) is used usually in the case with finite quark chemical potential, however, once it is put into a theory, then its Fierz transformations will be bound to induce some effects even in the case with zero quark chemical potential. In this paper we will research such effects on the vacuum of a given pure scalar $q-q$ coupling model. In fact, based on the converse Fierz transformation matrices (A.10) and (A.19) we may put 
down the Fierz rearranged $\mathcal{L}_{4\left(S_{q q}\right)}^{\bar{q} q}$ from $q-q$ channel to $\bar{q}-q$ channel and furthermore by using the transformation (A.8) obtain its exchange term $\mathcal{L}_{4\left(S_{q q}\right)}^{\bar{q} q-e x}$ which is in fact identical to $\mathcal{L}_{4\left(S_{q q}\right)}^{\bar{q} q}$. Thus the effective Lagrangian for $N_{f}=2$ becomes

$$
\begin{aligned}
& \mathcal{L}_{4\left(S_{q q)}\right)}^{e f f}=\mathcal{L}_{4\left(S_{q q}\right)}+\mathcal{L}_{4\left(S_{S q}\right)}^{\bar{q} q}+\mathcal{L}_{4\left(S_{q q}\right)}^{\bar{q} q-e x} \\
& =\mathcal{L}_{4\left(S_{q q}\right)}+G_{S}(\bar{q} q)^{2}+G_{P \tau}\left(\bar{q} i \gamma_{5} \tau_{a} q\right)^{2}+G_{V \lambda}\left(\bar{q} \gamma^{\mu} \lambda_{a} q\right)^{2}+\cdots \\
& G_{S}=G_{P \tau}=\left(N_{c}-1\right) H_{S} / 4 N_{c}, G_{V \lambda}=-H_{S} / 8
\end{aligned}
$$

Hence, as a result of the converse Fierz transformations, we are led from the pure scalar $q-q$ channel coupling (9) to the scalar and pseudoscalar isovector coupling $(\bar{q} q)^{2}$ and $\left(\bar{q} i \gamma_{5} \tau_{a} q\right)^{2}$. When $N_{f}=2$, they have the same coupling strengths $G_{S}=G_{P \tau}$ and this means that the chiral
$S U_{f L}(2) \otimes S U_{f R}(2)$ symmetry is maintained. Meantime, we are also led to the four fermion interactions similar to the ones induced by heavy gluon exchange, but with weaker strength $G_{V \lambda}=-H_{S} / 8$. It is interesting to make a comparison between the values of $G_{S}$ and $H_{S}$. For a given $G_{S}$ and $H_{S}$, equation (7) in [18] has given the possible least value points of the effective potential $V_{4}(\sigma,|\Delta|)$ (the ground states) of the model with the coupling terms corresponding to $G_{S}$ and $H_{S}$, where $\sigma$ and $\Delta$ represent the order parameters relevant to the scalar $\bar{q}-q$ condensates and the scalar $q-q$ condensates respectively. In present case, the induced $G_{S}$ depends on $H_{S}$ and $N_{c}$, and (7) in [18] will be reduced to the following form: the ground state of the model will be at

$$
(\sigma,|\Delta|)=\left\{\begin{array}{ccc}
\left(0, \Delta_{1}\right) \text { if } & 1 / 2<\tilde{H}_{S}<\left(3 N_{c}+1\right) /\left(N_{c}-1\right)\left(N_{c}-2\right), & N_{c}<9 \\
\left(\sigma_{2}, \Delta_{2}\right) \text { if } & \tilde{H}_{S}>\left(3 N_{c}+1\right) /\left(N_{c}-1\right)\left(N_{c}-2\right), & N_{c}<9 \\
\left(\sigma_{1}, 0\right) \text { if } & \tilde{H}_{S}>4 /\left(N_{c}-1\right), & N_{c}>9
\end{array}\right.
$$

where we have used the denotations $\tilde{H}_{S} \equiv H_{S} \Lambda^{2} / \pi^{2}$ and $\Lambda$ is the 4D Euclidean momentum cutoff of the loop integrals. It is indicated that $N_{c}<9$ and $N_{c}>9$ correspond respectively to $G_{S} / H_{S}<2 / N_{c}$ and $G_{S} / H_{S}>2 / N_{c}$. Hence, when $N_{c}<9$ i.e. $G_{S} / H_{S}<2 / N_{c}$, for a given $N_{c}$, the system can be in a pure $q-q$ condensate phase only if the coupling strength $H_{S}$ is less than the critical value $\left(3 N_{c}+1\right) /\left(N_{c}-1\right)\left(N_{c}-2\right)$. Especially, in the limit of $N_{c}=2$, the critical value of $H_{S}$ goes to $\infty$ and this implies that the system will only be in the chiral invariant pure $q-q$ condensate phase. Once $3 \leq N_{c}<9$ and $\tilde{H}_{S}$ exceeds the above critical value, the pure $q-q$ condensate phase will be changed into a coexistence phase with the $q-q$ and the $\bar{q}-q$ condensates, though the original purpose of our using $\mathcal{L}_{4\left(S_{q q}\right)}$ in (9) is only for expounding the pure $q q$ condensate. In particular, for the realistic case with $N_{c}=3$ of $\mathrm{QCD}$, the expected pure $q-q$ condensate phase could appear only if $\tilde{H}_{S}<5$. This is a very interesting result. Once the strength $\tilde{H}_{S}$ of the given $q-q$ channel coupling is large enough, what could emerge from the vacuum will no longer be the expected diquark condensates, instead, be a coexistence of the $q-q$ and $\bar{q}-q$ condensates. The critical value of $\tilde{H}_{S}$ will decrease as the increase of $N_{c}$, for example, it becomes $25 / 42$ for $N_{c}=8$. On the other hand, when $N_{c}>9$ i.e. $G_{S} / H_{S}>2 / N_{c}$, for a suffiently large
$\tilde{H}_{S}$, there could exist only the $\bar{q}-q$ condensates and no the $q-q$ condensates. This statement will certainly keep to be valid until $N_{c} \rightarrow \infty$, consistent with the general conclusion reached in [18]. The present key point lies in that even if the starting point (9) is a pure scalar $q-q$ channel coupling, as a result of the converse Fierz transformations, the above general conclusion is also true.

\section{2D Four Fermion Interactions}

\subsection{Scalar and Pseudoscalar Isovector Antiquark-quark Channel Couplings}

Similar to the $4 \mathrm{D}$ case, we take the Lagrangian by

$$
\mathcal{L}_{2(S+P \tau)}=G\left[(\bar{q} q)^{2}+\left(\bar{q} i \gamma_{5} \tau_{a} q\right)^{2}\right] .
$$

However, in 2D case, we need not to consider the continuous symmetries of a Lagrangian, since they can never be spontaneously broken based on Mermin-Wagner-Coleman theorem [23]. Formally (13) is the same as (1), but now the $\gamma_{5}$ in it is a $2 \times 2$ matrix. The steps to conduct the Fierz transformations are similar to the ones taken in $4 \mathrm{D}$ case in Section 2. Based on the Fierz transformations (A.11), (A.17), (A.12) and (A.18), the resulting total effective Lagrangian $\mathcal{L}_{2(S+P \tau)}^{e f f}$ for $N_{f}=2$ becomes

$$
\mathcal{L}_{2(S+P \tau)}^{e f f}=\mathcal{L}_{2(S+P \tau)}+\mathcal{L}_{2(S+P \tau)}^{e x}+\mathcal{L}_{2(S+P \tau)}^{q q}
$$

$$
\mathcal{L}_{2(S+P \tau)}^{e x}=\frac{G}{2 N_{c}}\left[(\bar{q} q)^{2}+\left(\bar{q} i \gamma_{5} \tau_{a} q\right)^{2}-\left(\bar{q} \tau_{a} q\right)^{2}-\left(\bar{q} i \gamma_{5} q\right)^{2}-2\left(\bar{q} \gamma^{\mu} q\right)^{2}-N_{c}\left(\bar{q} \gamma^{\mu} \lambda_{a^{\prime}} q\right)^{2}\right]+\frac{G}{4}\left\{\left[\left(\bar{q} i \gamma_{5} \tau_{a} \lambda_{a} q\right)^{2}-\left(\bar{q} i \gamma_{5} \lambda_{a^{\prime}} q\right)^{2}\right]-\left[i \gamma_{5} \rightarrow 1_{s}\right]\right\}
$$




$$
\mathcal{L}_{2(S+P \tau)}^{q q}=\frac{G}{4} \sum_{a^{\prime}=S^{\prime}, A^{\prime}}\left\{\left[\left(\bar{q} i \gamma_{5} \tau_{S} \lambda_{a^{\prime}} q^{c}\right)\left(\bar{q}^{c} i \gamma_{5} \tau_{S} \lambda_{a^{\prime}} q\right)-\left(\bar{q} i \gamma_{5} \tau_{A} \lambda_{a^{\prime}} q^{c}\right)\left(\bar{q}^{c} i \gamma_{5} \tau_{A} \lambda_{a^{\prime}} q\right)-\left(i \gamma_{5} \rightarrow 1_{s}\right)\right]-2\left(\bar{q} \gamma^{\mu} \tau_{A} \lambda_{a^{\prime}} q^{c}\right)\left(\bar{q}^{c} \gamma_{\mu} \tau_{A} \lambda_{a^{\prime}} q\right)\right\}
$$

where $\tau_{S}$ and $\lambda_{S^{\prime}}$ are respectively symmetric generators of $U_{f}\left(N_{f}\right)$ and $U_{c}\left(N_{c}\right)$, including $\tau_{0} \equiv \sqrt{2 / N_{f}} 1_{f}$ and $\lambda_{0} \equiv$ $\sqrt{2 / N_{c}} 1_{c}$. It is indicated that when $N_{f}=2$, the coupling terms $\left(\bar{q} \gamma^{\mu} \tau_{a} q\right)^{2}$ and $\left(\bar{q} \gamma^{\mu} \tau_{a} \lambda_{a} q\right)^{2}$ have disappeared. We see that in $\mathcal{L}_{2(S+P \tau)}^{e f f}$, the scalar and pseudoscalar isoscalar channel couplings $(\bar{q} q)^{2}$ and $\left(\bar{q} i \gamma_{5} \tau_{a} q\right)^{2}$ keep to be the maximal attractive ones. Denote the respective coupling strengths by $G_{S}$ and $G_{P \tau}$, then we will have $G_{S}=G_{P \tau}=\left(1+1 / 2 N_{c}\right) G$.

Consequently the model will maintain its original feature unchanged. On the other hand, the Fierz transformations have also led to occurrence of the scalar and pseudoscalar $q-q$ attractive channel couplings $\left(\bar{q} i \gamma_{5} \tau_{S} \lambda_{A^{\prime}} q^{c}\right)\left(\bar{q}^{c} i \gamma_{5} \tau_{S} \lambda_{A^{\prime}} q\right)$ and $\left(\bar{q} \tau_{A} \lambda_{A^{\prime}} q^{c}\right)\left(\bar{q}^{c} \tau_{A} \lambda_{A^{\prime}} q\right) \quad$ with the coupling strength $H_{S}=G / 4$. However, considering the ratio

$$
\begin{gathered}
G_{S} / H_{S}=2\left(2 N_{c}+1\right) / N_{c}>2 / N_{c} \\
\mathcal{L}_{2(V \lambda)}^{e x}=G_{S} \sum_{a=0}^{3}\left[\left(\bar{q} \tau_{a} q\right)^{2}+\left(\bar{q} i \gamma_{5} \tau_{a} q\right)^{2}\right]-\frac{g}{2 N_{c}} \sum_{a=0}^{3}\left[\left(\bar{q} \tau_{a} \lambda_{a^{\prime}} q\right)^{2}+\left(\bar{q} i \gamma_{5} \tau_{a} \lambda_{a^{\prime}} q\right)^{2}\right], \tau_{0}=1_{f}, G_{S}=\left(N_{c}^{2}-1\right) g / N_{c}^{2}
\end{gathered}
$$

we can affirm similarly based on the general criterion derived in [18] that for the 2D four fermion interaction model expressed by (13), only the antiquark-quark condensates, rather than the diquark condensates, are possible in its vacuum.

\subsection{Four Fermion Interactions from Heavy Gluon Exchange}

Take the Lagrangian to be

$$
\mathcal{L}_{2(V \lambda)}=-g\left(\bar{q} \gamma^{\mu} \lambda_{a} q\right)\left(\bar{q} \gamma_{\mu} \lambda_{a} q\right),
$$

where $\gamma^{\mu}$ are $2 \times 2$ matrices. After the Fierz transformations, the total effective Lagrangian $\mathcal{L}_{2(V \lambda)}^{\text {eff }}$ for $N_{f}=2$ can be expressed as follows.

$$
\mathcal{L}_{2(V \lambda)}^{e f f}=\mathcal{L}_{2(V \lambda)}+\mathcal{L}_{2(V \lambda)}^{e x}+\mathcal{L}_{2(V \lambda)}^{q q}
$$

with

and

$$
\begin{aligned}
& \mathcal{L}_{2(V \lambda)}^{q q}=-\left[\left(N_{c}-1\right) g / 2 N_{c}\right] \sum_{a=S, A}\left[\left(\bar{q} i \gamma_{5} \tau_{a} \lambda_{S^{\prime}} q^{c}\right)\left(\bar{q}^{c} i \gamma_{5} \tau_{a} \lambda_{S^{\prime}} q\right)+\left(i \gamma_{5} \rightarrow 1_{s}\right)\right] \\
& +H_{S} \sum_{a=S, A}\left[\left(\bar{q} i \gamma_{5} \tau_{a} \lambda_{A^{\prime}} q^{c}\right)\left(\bar{q}^{c} i \gamma_{5} \tau_{a} \lambda_{A} q\right)+\left(i \gamma_{5} \rightarrow 1_{s}\right)\right], \quad H_{S}=\left(N_{c}+1\right) g / 2 N_{c} .
\end{aligned}
$$

Since $H_{S}>0$, so the corresponding coupling terms are attractive. However, the ratio of the strengths of the scalar $\bar{q}-q$ channel coupling $(\bar{q} q)^{2}$ and the scalar $q-q$ channel coupling $\left(\bar{q} i \gamma_{5} \tau_{S} \lambda_{A^{\prime}} q^{c}\right)\left(\bar{q}^{c} i \gamma_{5} \tau_{S} \lambda_{A^{\prime}} q\right)$ is obtained to be

$$
G_{S} / H_{S}=2\left(N_{c}-1\right) / N_{c}>2 / N_{c} \text {, for } N_{c}>2 .
$$

Hence, if $N_{c} \geq 3$, there will be antiquar-kquark condensates alone in the vacuum[18]. Equation (22) is the same as (8) in the $4 \mathrm{D}$ case.

\subsection{Scalar Diquark Channel Interactions}

The Lagrangian is given by [18]

$$
\mathcal{L}_{2\left(S_{q q}\right)}^{e f f}=\mathcal{L}_{2\left(S_{q q}\right)}+\mathcal{L}_{2\left(S_{q q}\right)}^{\bar{q} q}+\mathcal{L}_{2\left(S_{q q}\right)}^{\bar{q} q-e x}=\mathcal{L}_{2\left(S_{q q}\right)}+H_{S} \sum_{\Gamma^{b}=1_{s}, i_{5}, \gamma^{\mu}}\left[\frac{3\left(N_{c}-1\right)}{2 N_{c}}\left(\bar{q} \Gamma^{b} q\right)^{2}-\frac{3}{4}\left(\bar{q} \Gamma^{b} \lambda_{a^{\prime}} q\right)^{2}+\frac{N_{c}-1}{2 N_{c}}\left(\bar{q} \Gamma^{b} \tau_{a} q\right)^{2}-\frac{1}{4}\left(\bar{q} \Gamma^{b} \tau_{a} \lambda_{a^{\prime}} q\right)^{2}\right]
$$

Equation (24) contains the induced scalar channel term $(\bar{q} q)^{2}$ and pseudoscalar channel term $\left(\bar{q} i \gamma_{5} \tau_{a} q\right)^{2}$ which respectively have the coupling strengths $G_{S}=3\left(N_{c}-1\right) H_{S}$

$$
\mathcal{L}_{2\left(S_{q q}\right)}=H_{S}\left(\bar{q} i \gamma_{5} \tau_{S} \lambda_{A^{\prime}} q^{c}\right)\left(\bar{q}^{c} i \gamma_{5} \tau_{S} \lambda_{A^{\prime}} q\right)
$$

It is indicated that (23) is different from (9) with $\tau_{S}$ having replaced $\tau_{A}$ in (9), because in $2 \mathrm{D}$ case the matrix $C \gamma_{5}$ is symmetric. We may use the converse matrices (A.13) and (A.19) in the Appendix to obtain the converse Fierz rearranged $\mathcal{L}_{2\left(S_{q q}\right)}^{\bar{q} q}$ and furthermore use the transformation (A.11) in the Appendix to get its exchange terms $\mathcal{L}_{2\left(S_{q q}\right)}^{\bar{q} q-e}=\mathcal{L}_{2\left(S_{q q}\right)}^{\bar{q} q}$, thus the total effective Lagrangian for $N_{f}=2$ becomes
$2 N_{c}$ and $G_{P \tau}=\left(N_{c}-1\right) H_{S} / 2 N_{c}$ and it may be seen that, among all the $\bar{q}-q$ channel couplings of $\mathcal{L}_{2\left(S_{q q}\right)}^{e f f}$, the scalar channel term $(\bar{q} q)^{2}$ is maximal attractive. We note that the 
ratio of $G_{S}$ and the strength $H_{S}$ of the scalar $q-q$ channel coupling i.e. $\mathcal{L}_{2\left(S_{q q}\right)}$ becomes

$$
G_{S} / H_{S}=\left[3\left(N_{c}-1\right) / 4\right]\left(2 / N_{c}\right) .
$$

Based on the general criterion given in [18], if there exist the $\bar{q}-q$ condensates alone in the vacuum, then the condition $G_{S} / H_{S}>2 / N_{c}$ must be satisfied, and from (25), this implies that $3\left(N_{c}-1\right) / 4>1$ and it leads to $N_{c}>7 / 3$. Therefore, if $N_{c} \geq 3$, the vacuum of the system will in fact only be in a $\bar{q}-q$ condensate phase, even though the originally given interaction (23) is a pure scalar $q-q$ channel coupling. On the other hand, if $N_{c}=2<7 / 3$, we will have $G_{S} / H_{S}<2 / N_{c}$, however, owing to $G_{S} \neq 0$, theoretically one could just acquire a mixed phase with both $\bar{q}-q$ and $q-q$ condensates, since in 2D case, it was proven that one could get pure $q-q$ condensate phase in the vacuum only if $G_{S}=0$ [18].

\section{3D Four Fermion Interactions}

\subsection{Scalar and Isovector Antiquark-Quark Channel Couplings}

Since there is not $\gamma_{5}$ matrix in $3 \mathrm{D}$ spacetime, the

$$
\begin{aligned}
& \mathcal{L}_{3(S+S \tau)}^{e f f}=\mathcal{L}_{3(S+S \tau)}+\mathcal{L}_{3(S+S \tau)}^{e x}+\mathcal{L}_{3(S+S \tau)}^{q q}=G_{S}(\bar{q} q)^{2}+G_{S \tau}\left(\bar{q} \tau_{a} q\right)^{2}+H_{P}\left(\bar{q} \tau_{A} \lambda_{A^{\prime}} q^{c}\right)\left(\bar{q}^{c} \tau_{A} \lambda_{A^{\prime}} q\right)+\cdots, \\
& G_{S}=\left(1-1 / N_{c}\right) G, G_{S \tau}=G, H_{P}=G / 4 .
\end{aligned}
$$

It should be indicated that, after the Fierz transformations, two maximal attractive channel couplings are still the terms $(\bar{q} q)^{2}$ and $\left(\bar{q} \tau_{a} q\right)^{2}$ contained in the original $\mathcal{L}_{3(S+S \tau)}$. However, the two terms with the same coupling constant $G$ in $\mathcal{L}_{3(S+S \tau)}$ now have different coupling strengths $G_{S}<G_{S \tau}$. This implies that in the resulting $\mathcal{L}_{3(S+S \tau)}^{\text {eff }}$ the maximal attractive channel coupling will actually be the term $\left(\bar{q} \tau_{a} q\right)^{2}$ rather than the term $(\bar{q} q)^{2}$. So it is more reasonable to assume that the condensates $\left\langle\bar{q} \tau_{a} q\right\rangle$ are formed more easily than the condensates $\langle\bar{q} q\rangle$, and this will lead to spontaneous breaking of the flavor $S U_{f}\left(N_{f}\right)$ (for $N_{f}=2$ i.e. isospin) symmetry. In this case we must replace the order parameter $\sigma=-2 G_{S}\langle\bar{q} q\rangle$ by $\sigma=-2 G_{S \tau}\left\langle\bar{q} \tau_{3} q\right\rangle$ (it is possible to fix the condensates in the $\tau_{3}$ direction through a rotation in isospin space). However, it may be proven that the derived expression for the effective potential of the model containing the new $\sigma$ will keep unchanged in form, hence the conclusions reached in [18] about interplay between the $\bar{q}-q$ and $q-q$ condensates in the ground state (vacuum) is still true, the mere change is to replace the scalar channel coupling constant $G_{S}$ by the scalar similarities of (1) and (13) in 4D and 2D case will be the Lagrangian expressed by

$$
\mathcal{L}_{3(S+S \tau)}=G\left[(\bar{q} q)^{2}+\left(\bar{q} \tau_{a} q\right)^{2}\right]
$$

which is $S U_{c}\left(N_{c}\right) \otimes S U_{f}\left(N_{f}\right) \otimes U_{f}(1)$ invariant. For convenience, the coupling strengths of the two terms in $\mathcal{L}_{3(S+S \tau)}$ are assumed to be equal, but physically this is not essential. When $N_{f}=2$, by (A.14) and (A.17), the Fierz rearranged

$$
\begin{aligned}
\mathcal{L}_{3(S+S \tau)}^{e x}= & -\frac{G}{N_{c}}\left[(\bar{q} q)^{2}+\left(\bar{q} \gamma^{\mu} q\right)^{2}\right] \\
& -\frac{G}{2}\left[\left(\bar{q} \lambda_{a^{\prime}} q\right)^{2}+\left(\bar{q} \gamma^{u} \lambda_{a^{\prime}} q\right)^{2}\right]
\end{aligned}
$$

and by (A.15) and (A.18), the Fierz rearranged

$$
\begin{gathered}
\mathcal{L}_{3(S+S \tau)}^{q q}=\frac{G}{4} \sum_{a^{\prime}=S^{\prime}, A^{\prime}}\left\{\left[\left(\bar{q} \tau_{A} \lambda_{a^{\prime}} q^{c}\right)\left(\bar{q}^{c} \tau_{A} \lambda_{a^{\prime}} q\right)+\left(1_{s} \rightarrow \gamma^{\mu}\right)\right]\right. \\
\left.-\left[\left(\bar{q} \tau_{S} \lambda_{a^{\prime}} q^{c}\right)\left(\bar{q}^{c} \tau_{S} \lambda_{a^{\prime}} q\right)+\left(1_{s} \rightarrow \gamma^{\mu}\right)\right]\right\}
\end{gathered}
$$

Thus the total effective Lagrangian becomes

isovector channel constant $G_{S \tau}$.

Since

$$
G_{S \tau} / H_{P}=4>2 / N_{c},
$$

we can immediately conclude that although the Fierz transformations may bring about the $q-q$ channel coupling corresponding to $H_{P}$, it is still impossible to form the pseudoscalar diquark condensates $\left\langle\bar{q} \tau_{A} \lambda_{A^{\prime}} q^{c}\right\rangle$ in the vacuum and the vacuum could only be in the $\left\langle\bar{q} \tau_{3} q\right\rangle$ condenstate phase.

\subsection{Four Fermion Interactions from Heavy Gluon Exchange}

The Lagrangian is given by

$$
{ }_{3(V \lambda)}=-g\left(\bar{q} \gamma^{\mu} \lambda_{a} q\right)\left(\bar{q} \gamma_{\mu} \lambda_{a} q\right)
$$

where $\gamma^{\mu}$ is $2 \times 2$ matrices in $3 \mathrm{D}$ spacetime. When $N_{f}=2$, the Fierz rearranged 


$$
\begin{aligned}
& \mathcal{L}_{3(V \lambda)}^{e x}=\sum_{a=0}^{3}\left[G_{S}\left(\bar{q} \tau_{a} q\right)^{2}-\frac{3 g}{4 N_{c}}\left(\bar{q} \tau_{a} \lambda_{a^{\prime}} q\right)^{2}-\frac{N_{c}^{2}-1}{2 N_{c}^{2}} g\left(\bar{q} \gamma^{\mu} \tau_{a} q\right)^{2}+\frac{g}{4 N_{c}}\left(\bar{q} \gamma^{\mu} \tau_{a} \lambda_{a^{\prime}} q\right)^{2}\right] \\
& G_{S}=3\left(N_{c}^{2}-1\right) g / 2 N_{c}^{2},
\end{aligned}
$$

and

$$
\begin{aligned}
& \mathcal{L}_{3(V \lambda)}^{q q}=H_{P}\left(\bar{q} \tau_{A} \lambda_{A^{\prime}} q^{c}\right)\left(\bar{q}^{c} \tau_{A} \lambda_{A^{\prime}} q\right)-\frac{3\left(N_{c}-1\right)}{4 N_{c}} g\left(\bar{q} \tau_{A} \lambda_{S^{\prime}} q^{c}\right)\left(\bar{q}^{c} \tau_{A} \lambda_{S^{\prime}} q\right)-\frac{N_{c}+1}{4 N_{c}} g\left(\bar{q} \gamma^{\mu} \tau_{A} \lambda_{A^{\prime}} q^{c}\right)\left(\bar{q}^{c} \gamma_{\mu} \tau_{A} \lambda_{A^{\prime}} q\right) \\
& +\frac{N_{c}-1}{4 N_{c}} g\left(\bar{q} \gamma^{\mu} \tau_{A} \lambda_{S^{\prime}} q^{c}\right)\left(\bar{q}^{c} \gamma_{\mu} \tau_{A} \lambda_{S^{\prime}} q\right)+\left(\tau_{A} \rightarrow \tau_{S}\right), \\
& \quad H_{P}=3\left(N_{c}+1\right) g / 4 N_{c} .
\end{aligned}
$$

Thus the total effective Lagrangian becomes

$$
\begin{aligned}
\mathcal{L}_{3(V \lambda)}^{e f f}= & \mathcal{L}_{3(V \lambda)}+\mathcal{L}_{3(V \lambda)}^{e x}+\mathcal{L}_{3(V \lambda)}^{q q} \\
= & G_{S}\left[(\bar{q} q)^{2}+\left(\bar{q} \tau_{a} q\right)^{2}\right]+H_{P}\left(\bar{q} \tau_{A^{\prime}} \lambda_{A^{\prime}} q^{c}\right)\left(\bar{q}^{c} \tau_{A} \lambda_{A^{\prime}} q\right) \\
& -\left(1-1 / 4 N_{c}\right) g\left(\bar{q} \gamma^{\mu} \lambda_{a^{\prime}} q\right)^{2}+\cdots .
\end{aligned}
$$

Since the ratio

$$
G_{S} / H_{P}=2\left(N_{c}-1\right) / N_{c}>2 / N_{c}, \text { for } N_{c}>2,
$$

we can assert that there could not be the diquark condensates in the vacuum of the model if $N_{c} \geq 3$ [18].
The corresponding Lagrangian is given by

$$
\mathcal{L}_{3\left(P_{q q}\right)}=H_{P}\left(\bar{q} \tau_{A} \lambda_{A^{\prime}} q^{c}\right)\left(\bar{q}^{c} \tau_{A} \lambda_{A^{\prime}} q\right)
$$

By using the converse matrices (A.16) and (A.19) in the Appendix, we may obtain the Fierz rearranged form $\mathcal{L}_{3\left(P_{q q}\right)}^{\bar{q} q}$ of (31) from $q-q$ channel to $\bar{q}-q$ channel, and then by (A.14) in the Appendix get its exchange terms $\mathcal{L}_{3\left(P_{q q}\right)}^{\bar{q} q-e x}=\mathcal{L}_{3\left(P_{q q}\right)}^{\bar{q} q}$, thus when $N_{f}=2$, their sum becomes

\subsection{Pseudoscalar Diquark Channel Coupling}

$$
\mathcal{L}_{3\left(P_{q q}\right)}^{\bar{q} q}+\mathcal{L}_{3\left(P_{q q}\right)}^{\bar{q} q-e x}=\frac{N_{c}-1}{2 N_{c}} H_{P}\left[\left(\bar{q} \tau_{a} q\right)^{2}-(\bar{q} q)^{2}\right]+\frac{H_{P}}{4}\left[\left(\bar{q} \lambda_{a^{\prime}} q\right)^{2}-\left(\bar{q} \tau_{a} \lambda_{a^{\prime}} q\right)^{2}\right]-\left(1_{s} \rightarrow \gamma^{\mu}\right)=G_{S \tau}\left(\bar{q} \tau_{a} q\right)^{2}+G_{S}(\bar{q} q)^{2}+\cdots,
$$

where $G_{S \tau}=\left(N_{c}-1\right) H_{P} / 2 N_{c}=-G_{S}>0$, this implies that only the term $\left(\bar{q} \tau_{a} q\right)^{2}$ is a (maximally) attractive interaction which could induce the isovector condensates $\left\langle\bar{q} \tau_{a} q\right\rangle$. As has been indicated in the sector of scalar and isovector $\bar{q}-q$ channel couplings, making the substitutions $\langle\bar{q} q\rangle \rightarrow\left\langle\bar{q} \tau_{a} q\right\rangle$ and $G_{S} \rightarrow G_{S \tau}$, we can conduct the same discussions and reach the same conclusions as the ones obtained in [18, 24 ] about interplay between the $\bar{q}-q$ and $q-q$ condensates. A special feature is now that the induced coupling constant $G_{S \tau}$ depends on $H_{P}$ and $N_{c}$. Let $\sigma=-2 G_{S \tau}\left\langle\bar{q} \tau_{3} q\right\rangle$ and $\Delta$ represent the order parameters respectively corresponding to the $\bar{q}-q$ and $q-q$ condensates, then the conclusion (34) in $[18,24]$ will be reduced to the following equation which shows the least value points of the effective potential $V_{3}(\sigma,|\Delta|)$ being at

$$
(\sigma,|\Delta|)=\left\{\begin{array}{llll}
\left(0, \Delta_{1}\right), & \text { if } & \tilde{H}_{P}>1 / 8 & N_{c} \leq 4<5 \\
\left(\sigma_{1}, 0\right), & \text { if } & \tilde{H}_{P}>1 / 2\left(N_{c}-1\right), & N_{c}>5
\end{array}\right.
$$

where $\tilde{H}_{P} \equiv H_{P} \Lambda_{3} / \pi^{2}, \Lambda_{3}$ is the $3 \mathrm{D}$ Euclidean momentum cutoff of the loop integrals. It is indicated that $N_{c}=5$ corresponds to $G_{S \tau} / H_{P}=2 / N_{c}$. We see from (33) that the four fermion interactions used to describe pure pseudoscalar diquark condensates, after the converse Fierz transformations, will induce the $\bar{q}-q$ channel coupling term $\left(\bar{q} \tau_{a} q\right)^{2}$ and lead to interplay between the $\bar{q}-q$ and $q-q$ condensates in the ground state. In this model, besides enough coupling strength $\tilde{H}_{P}$, the $q-q$ condensates could be formed only if $N_{c} \leq 4<5$ and in that case the ground state could be in a pure $q-q$ condensate phase; however, once $N_{c}>5$ and untill $N_{c} \rightarrow \infty$, we will be able to get merely the $\bar{q}-q$ condensates $\left\langle\bar{q} \tau_{a} q\right\rangle$ instead of the diquark condensates.

\section{Conclusions}

In this paper, we have theoretically analyzed possible effects of the Fierz transformations on the vacua of several given typical 4D, 2D and 3D two flavor and $N_{c}$ color four 
fermion interaction models. The results can be summarized as follows.

It is shown that after the Fierz transformations, the 4D and 2D scalar and pseudoscalar isovector couplings keep to be the maximal attractive ones with the strength $G_{S}$, and some scalar diquark channel couplings with the strength $H_{S}$ will be induced; in the case of 3D scalar and isovector coupling with equal strength, the isovector coupling will become the maximal attractive one with the strength $G_{S \tau}$ and one also gets the induced pseudoscalar $q-q$ channel coupling with the strength $H_{P}$. However, it is found that the resulting ratios $G_{S} / H_{S}$ and $G_{S \tau} / H_{P}$ are both always greater than the critical value $2 / N_{c}$. This indicates that no diquark condensates could be generated in the vacua of these models, hence the above interaction models will maintain to be the ones merely to describe possible $\bar{q}-q$ condensates. The above results are valid for any $N_{c}$.

For the four fermion interactions from heavy gluon exchange, no matter in 4D or 2D or 3D case, after the Fierz transformations, we will always get the ratio of the induced scalar $\bar{q}-q$ channel coupling strength $G_{S}$ and the induced $4 \mathrm{D}$ and $2 \mathrm{D}$ scalar or 3D pseudoscalar $q-q$ channel coupling strength $H_{S}$ or $H_{P}$ expressed by $G_{S} / H_{S}=G_{S} / H_{P}=2\left(N_{c}-1\right) / N_{c}$.

On the same ground as the above, if $N_{c} \geq 3$, this removes the possibility to emerge the diquarnk condensates from the vacua and only the $\bar{q}-q$ condensates could exist in the vacua. When the starting points are the pure diquark scalar or pseudoscalar channel couplings with the strengths $H_{S}$ or $H_{P}$, the nontrivial effects of the Fierz transformations on the vacua will be displayed. Owing to the converse Fierz transformations, we will get the induced $\bar{q}-q$ channel couplings including the exchange terms with the strength $G_{S}$ or $G_{S \tau}$ and this is bound to lead to interplay between the $\bar{q}-q$ and $q-q$ condensates. We have found that, independent of dimensionality of spacetime, the expected $q-q$ condensates could emerge from the vacua only if $N_{c}<N_{c}^{0}$, a critical value determined by the conditions $G_{S} / H_{S}<2 / N_{c}$ or $G_{S} / H_{P}$ $<2 / N_{c}$ which is $9,7 / 3$ and 5 for $4 \mathrm{D}, 2 \mathrm{D}$ and $3 \mathrm{D}$ case respectively.

In $4 \mathrm{D}$ case, when $N_{c}<9$, only if the coupling strength $H_{S}$ is less than some $N_{c}$ dependent critical value, we may get a pure $q-q$ condensate phase, otherwise, we will obtain a coexistence phase with the $q-q$ and $\bar{q}-q$ condensates. For the realistic case of $N_{c}=3$, the above critical value of $H_{S}$ corresponds to $H_{S} \Lambda^{2} / \pi^{2}=5$. Hence, owing to the Fierz transformations, a sufficiently strong $q-q$ channel coupling could lead to not the expected pure $q-q$ condensates, instead only a coexistence of the $q-q$ and $\bar{q}-q$ condensates in the vacuum. In $2 \mathrm{D}$ case, even if $N_{c}<7 / 3$, we can only obtain a coexistence phase with the two condensates. In 3D case, only under the condition $N_{c} \leq 4<5$ we may have a pure $q$ - $q$ condensate phase.

Once $N_{c}>N_{c}^{0}$, until $N_{c} \rightarrow \infty$, in all cases we will obtain only the $\bar{q}-q$ condensates and no the $q-q$ condensates in the vacua, though the original purpose to introduce the pure $q-q$ channel couplings is to deal with the diquark condensates.

The above results show that for the models which originally do not contain the diquark channel couplings, it seems to be unnecessary for us to worry about the occurrence of the diquark condensates in the vacua through the Fierz transformations. However, for a model of given diquark channel couplings, for example, in 4D spacetime, one must note that the expected pure diquark condensates could appear only in the case of weak coupling and some small $N_{c}$, and this is just the nontrivial effect of the Fierz transformations on the model's vacuum to which now one must pay special attention. In addition, if one attempts to extend the above analysis based on the mean field approximation to higher order correction of the $1 / N_{c}$ expansion, then because the above effects induced by the Fierz transformations depend on the value of $N_{c}$, more careful consideration must be conducted.

The analysis made in this paper can be extended to the case of finite temperature and finite quark chemical potential where the Fierz transformations will lead to the interplay between the thermal $\bar{q}-q$ and $q-q$ condensates which could or could not affect the feature of the ground state of a thermal four fermion interaction model.

In this paper, we only research some special 2 flavor and $N_{c}$ color four fermion models, however, the discussions may be of more general significance for any 2 flavor and $N_{c}$ color four fermion model, since for any given such model, the Fierz transformations can always lead to scalar $\bar{q}-q$ and scalar or pseudoscalar $q-q$ channel coupling and induce the interplay between the corresponding condensates in the ground state. In addition, similar or possibly different effects may be assumed to emerge from more general four fermion interaction models with dynamical symmetry breaking. It is just the above research which, for the first time, connects the Fierz transformations with the ground states of a class of four fermion interaction models with dynamical symmetry breaking thus provides us a new angle of view to inspect this class of models. In any case, theoretically, the possible ground state effects of the Fierz transformations should become an implicit factor to build and treat such class of models.

\section{Appendix: Fierz Transformations}

The Fierz transformations in Dirac spinor space of 4D spacetime and in $U(N)$ space can be found in Appendix A of [13]. However, for this paper to be selfcontained and convenience of use, we will still give a brief introduction of the Fierz transformations and list all the necessary explicit expressions for the transformations, including the new results 
in spinor space of $2 \mathrm{D}$ and $3 \mathrm{D}$ spacetime and the converse forms of all the nonselfconverse Fierz transformations.

Consider a local four fermion interaction of spinor fields $q \equiv q(x)$ with $N_{f}$ flavors and $N_{c}$ colors, the corresponding Lagrangian is

$$
\mathcal{L}_{\text {int }}=g\left(\bar{q} \Gamma^{a} q\right)^{2}=g \Gamma_{12}^{a} \Gamma_{34}^{a} \bar{q}_{1} q_{2} \bar{q}_{3} q_{4},
$$

where $g$ is the coupling constant, $\Gamma^{a}$ is outer product of the linearly independent matrices in spinor, flavor $U_{f}\left(N_{f}\right)$ and color $U_{c}\left(N_{c}\right)$ space, the numbers 1, 2, 3 and 4 represent the indices of the elements of $\Gamma^{a}$, for instance, the number 1 can represent $s_{1} f_{1} c_{1}$ for the product matrix, or $s_{1}, f_{1}$ and $c_{1}$ when $\Gamma^{a}$ is separately limited to the matrix acting on spin, flavor and color space etc. and an index repeated always means its summing. In view of anticommutativity of the fermion fields $q$, (A.1) may be rewritten by

$$
\mathcal{L}_{\text {int }}=-g \Gamma_{12}^{a} \Gamma_{34}^{a} \bar{q}_{1} q_{4} \bar{q}_{3} q_{2}=: \mathcal{L}_{\text {int }}^{e x}
$$

or

$$
\mathcal{L}_{\text {int }}=g \Gamma_{12}^{a} \Gamma_{34}^{a} \bar{q}_{1} \bar{q}_{3} q_{4} q_{2}=: \mathcal{L}_{\text {int }}^{q q}
$$

In the above expressions, we will restrict ourselves to Hartree-type approximation, for example, in (A.2), $\bar{q}_{1}$ is contracted with $q_{4}$ and $\bar{q}_{3}$ is contracted with $q_{2}$ thus $\mathcal{L}_{\text {int }}^{e x}$ will give exchange diagram of $\mathcal{L}_{\text {int }}$, and in (A.3), $\bar{q}_{1}$ is contracted with $\bar{q}_{3}$ and $q_{4}$ is contracted with $q_{2}$ thus $\mathcal{L}_{\text {int }}^{q q}$ will give the coupling term of antiquark-antiquark $(\bar{q}-\bar{q})$ and quark-quark $(q-q)$. For this purpose, in (A.2) we must rewrite the matrices

$$
\Gamma_{12}^{a} \Gamma_{34}^{a}=\sum_{b} c_{b}^{a} \Gamma_{14}^{b} \Gamma_{32}^{b},
$$

Where $b$ runs over all the linearly independent matrices $\Gamma^{b}$.
In this paper, (A.4) will be called the Fierz transformation of the $\bar{q}-q \rightarrow \bar{q}-q$ channel. By means of (A.4), (A.2) becomes

$$
\mathcal{L}_{\text {int }}^{e x}=-g \sum_{b} c_{b}^{a}\left(\bar{q} \Gamma^{b} q\right)^{2} .
$$

On the other hand, in (A.3) we must rewrite the matrices

$$
\Gamma_{12}^{a} \Gamma_{34}^{a}=\sum_{b} d_{b}^{a}\left(\Gamma^{b} C\right)_{13}\left(C \Gamma^{b}\right)_{42},
$$

where $C$ is the charge conjugate matrix in spinor space. Equation (A.6) will be called the Fierz transformation of the $\bar{q}-q \rightarrow q-q$ channel. By means of (A.6), (A.3) becomes

$$
\mathcal{L}_{\text {int }}^{q q}=g \sum_{b} d_{b}^{a}\left(\bar{q} \Gamma^{b} q^{c}\right)\left(\bar{q}^{c} \Gamma^{b} q\right)
$$

where $q^{c}=C \bar{q}^{\mathrm{T}}$ and $\bar{q}^{c}=q^{\mathrm{T}} C$ are charge conjugates of the fields $q$ and $\bar{q}$ respectively.

The problem to solve the Fierz transformations is reduced to calculate the expansion coefficients $c_{b}^{a}$ and $d_{b}^{a}$ in (A.4) and (A.6). In view of the outer product feature of $\Gamma^{a}$ and the similarity of the group $U_{f}\left(N_{f}\right)$ and $U_{c}\left(N_{c}\right)$, we can consider separately the cases of that $\Gamma^{a}$ are the matrices in spinor space and that $\Gamma^{a}=\left\{1, \tau_{a}\right\}$ with 1 as the unit matrix and $\tau a(a=1, \cdots, N-1)$ as the generators of the group $S U(N)$.

In the following we will give explicit expressions of the Fierz transformations, of the matrices in spinor spaces in 4D, $2 \mathrm{D}$ and $3 \mathrm{D}$ spacetime and of the $U(N)$ generators.

\section{A.1. Matrices in Spinor Space}

\section{A.1.1. 4D Spacetime}

The independent $4 \times 4$ Dirac matrices are $1_{s}, i \gamma_{5}, \gamma^{\mu}, \gamma^{\mu} \gamma_{5}, \sigma^{\mu v}(\mu, \nu=0,1,2,3)$.

The Fierz tranformationtions become

$$
\left(\begin{array}{c}
\left(1_{s}\right)_{12}\left(1_{s}\right)_{34} \\
\left(i \gamma_{5}\right)_{12}\left(i \gamma_{5}\right)_{34} \\
\left(\gamma^{\mu}\right)_{12}\left(\gamma_{\mu}\right)_{34} \\
\left(\gamma^{\mu} \gamma_{5}\right)_{12}\left(\gamma_{\mu} \gamma_{5}\right)_{34} \\
\left(\sigma^{\mu \nu}\right)_{12}\left(\sigma_{\mu \nu}\right)_{34}
\end{array}\right)=\underbrace{\left(\begin{array}{rrrrr}
\frac{1}{4} & -\frac{1}{4} & \frac{1}{4} & -\frac{1}{4} & \frac{1}{8} \\
-\frac{1}{4} & \frac{1}{4} & \frac{1}{4} & -\frac{1}{4} & -\frac{1}{8} \\
1 & 1 & -\frac{1}{2} & -\frac{1}{2} & 0 \\
-1 & -1 & -\frac{1}{2} & -\frac{1}{2} & 0 \\
3 & -3 & 0 & 0 & -\frac{1}{2}
\end{array}\right)}_{F_{\bar{q} q \rightarrow \bar{q} q}^{s_{4}}}\left(\begin{array}{c}
\left(1_{s}\right)_{14}\left(1_{s}\right)_{32} \\
\left(i \gamma_{5}\right)_{14}\left(i \gamma_{5}\right)_{32} \\
\left(\gamma^{\mu}\right)_{14}\left(\gamma_{\mu}\right)_{32} \\
\left(\gamma^{\mu} \gamma_{5}\right)_{14}\left(\gamma_{\mu} \gamma_{5}\right)_{32} \\
\left(\sigma^{\mu \nu}\right)_{14}\left(\sigma_{\mu \nu}\right)_{32}
\end{array}\right) \quad(\bar{q}-q \rightarrow \bar{q}-q \text { channel })
$$




$$
=\underbrace{\left(\begin{array}{rrrrr}
\frac{1}{4} & -\frac{1}{4} & \frac{1}{4} & -\frac{1}{4} & -\frac{1}{8} \\
-\frac{1}{4} & \frac{1}{4} & \frac{1}{4} & -\frac{1}{4} & \frac{1}{8} \\
1 & 1 & -\frac{1}{2} & -\frac{1}{2} & 0 \\
1 & 1 & \frac{1}{2} & \frac{1}{2} & 0 \\
-3 & 3 & 0 & 0 & -\frac{1}{2}
\end{array}\right)}_{F_{\bar{q} q \rightarrow q q}^{S_{4}}}\left(\begin{array}{c}
\left(i \gamma_{5} C\right)_{13}\left(C i \gamma_{5}\right)_{42} \\
(C)_{13}(C)_{42} \\
\left(\gamma^{\mu} \gamma_{5} C\right)_{13}\left(C \gamma_{\mu} \gamma_{5}\right)_{42} \\
\left(\gamma^{\mu} C\right)_{13}\left(C \gamma_{\mu}\right)_{42} \\
\left(\sigma^{\mu v} C\right)_{13}\left(C \sigma_{\mu \nu}\right)_{42}
\end{array}\right) \quad(\bar{q}-q \rightarrow q-q \text { channel })
$$

We note that the matrix $F_{\bar{q} q \rightarrow \bar{q} q}^{s_{4}}$ is selfconverse, i.e. $\left(F_{\bar{q} q \rightarrow \bar{q} q}^{s_{4}}\right)^{2}=1_{s}$, thus for the $\bar{q}-q \rightarrow \bar{q}-q$ channel, the positive and the converse Fierz transformations are identical. However, the same conclusion is not true for the $\bar{q}-q \rightarrow q-q$ channel. In fact, the converse of the matrix $F_{\bar{q} q \rightarrow q q}^{s_{4}}$ is

$$
\left(F_{\bar{q} q \rightarrow q q}^{S_{4}}\right)^{-1} \equiv F_{q q \rightarrow \bar{q} q}^{S_{4}}=\left(\begin{array}{rrrrr}
1 / 4 & -1 / 4 & 1 / 4 & 1 / 4 & -1 / 8 \\
-1 / 4 & 1 / 4 & 1 / 4 & 1 / 4 & 1 / 8 \\
1 & 1 & -1 / 2 & 1 / 2 & 0 \\
-1 & -1 & -1 / 2 & 1 / 2 & 0 \\
-3 & 3 & 0 & 0 & -1 / 2
\end{array}\right)
$$

Obviously, $\left(F_{\bar{q} q \rightarrow q q}^{s_{4}}\right)^{-1} \neq F_{\bar{q} q \rightarrow q q^{\circ}}^{s_{4}}$

\section{A.1.2. 2D Spacetime}

The independent $2 \times 2$ matrices in spinor space are $1_{s}, \gamma^{\mu}(\mu=0,1), \gamma_{5}=\gamma^{0} \gamma^{1}$, and the charge conjugate matrix $C=-\gamma^{1}$. One adoption of $\gamma^{\mu}$ is that $\gamma^{0}=\sigma^{3}, \gamma^{1}=i \sigma^{2}$ with Pauli matrices $\sigma^{i} \quad(i=1,2,3)$. The Fierz transformations become

which corresponds to the converse of the transformation (A.9).

$$
\begin{aligned}
& \left(\begin{array}{c}
\left(1_{s}\right)_{12}\left(1_{s}\right)_{34} \\
\left(i \gamma_{5}\right)_{12}\left(i \gamma_{5}\right)_{34} \\
\left(\gamma^{\mu}\right)_{12}\left(\gamma_{\mu}\right)_{34}
\end{array}\right)=\underbrace{\left(\begin{array}{ccc}
\frac{1}{2} & -\frac{1}{2} & \frac{1}{2} \\
-\frac{1}{2} & \frac{1}{2} & \frac{1}{2} \\
1 & 1 & 0
\end{array}\right)\left(\begin{array}{c}
\left(1_{s}\right)_{14}\left(1_{s}\right)_{32} \\
\left(i \gamma_{5}\right)_{14}\left(i \gamma_{5}\right)_{32} \\
\left(\gamma^{\mu}\right)_{14}\left(\gamma_{\mu}\right)_{32}
\end{array}\right) \quad(\bar{q}-q \rightarrow \bar{q}-q \text { channel })}_{F_{\bar{q} q \rightarrow \bar{q} q}^{s_{2}}} \\
& =\underbrace{\left(\begin{array}{ccc}
\frac{1}{2} & -\frac{1}{2} & -\frac{1}{2} \\
\frac{1}{2} & -\frac{1}{2} & \frac{1}{2} \\
1 & 1 & 0
\end{array}\right)}_{F_{\bar{q} q \rightarrow q q}^{s_{2}}}\left(\begin{array}{c}
\left(i \gamma_{5} C\right)_{13}\left(C i \gamma_{5}\right)_{42} \\
(C)_{13}(C)_{42} \\
\left(\gamma^{\mu} C\right)_{13}\left(C \gamma_{\mu}\right)_{42}
\end{array}\right) \quad(\bar{q}-q \rightarrow q-q \text { channel })
\end{aligned}
$$

It is also indicated that $F_{\bar{q} q \rightarrow \bar{q} q}^{S_{2}}=\left(F_{\bar{q} q \rightarrow \bar{q} q}^{S_{2}}\right)^{-1}$ is selfconverse, but $F_{\bar{q} q \rightarrow q q}^{S_{2}}$ is not, the latter's converse is

$$
\begin{aligned}
& \left(F_{\bar{q} q \rightarrow q q}^{s_{2}}\right)^{-1} \equiv F_{q q \rightarrow \bar{q} q}^{s_{2}}=\left(\begin{array}{ccc}
\frac{1}{2} & \frac{1}{2} & \frac{1}{2} \\
-\frac{1}{2} & -\frac{1}{2} & \frac{1}{2} \\
-1 & 1 & 0
\end{array}\right) \quad\left(\begin{array}{ll}
\text { A.13) } \\
\left(1_{s}\right)_{12}\left(1_{s}\right)_{34} \\
\left(\gamma^{\mu}\right)_{12}\left(\gamma_{\mu}\right)_{34}
\end{array}\right)=\underbrace{\left(\begin{array}{cc}
\frac{1}{2} & \frac{1}{2} \\
\frac{3}{2} & -\frac{1}{2}
\end{array}\right)}_{F_{\bar{q} q \rightarrow \bar{q} q}^{s_{3}}}\left(\begin{array}{l}
\left(1_{s}\right)_{14}\left(1_{S}\right)_{32} \\
\left(\gamma^{\mu}\right)_{14}\left(\gamma_{\mu}\right)_{32}
\end{array}\right)
\end{aligned}
$$

which leads to the converse of the transformation (A.12).

\section{A.1.3. 3D Spacetime}

The independent $2 \times 2$ matrices in spinor space are $1_{s}, \gamma^{\mu}(\mu=0,1,2)$ and the charge conjugate matrix $C=\gamma^{2}$, but no $\gamma_{5}$ exists. One adoption of $\gamma^{\mu}$ is that $\gamma^{0}=\sigma^{3}$, $\gamma^{1}=i \sigma^{1}, \gamma^{2}=i \sigma^{2}$ with the Pauli matrices $\sigma^{i}(i=1,2,3)$. The Fierz transformations are

$(\bar{q}-q \rightarrow \bar{q}-q$ channel $)$ 


$$
=\underbrace{\left(\begin{array}{cr}
-\frac{1}{2} & -\frac{1}{2} \\
\frac{3}{2} & -\frac{1}{2}
\end{array}\right)}_{F_{\bar{q} q \rightarrow q q}^{S_{3}}}\left(\begin{array}{c}
(C)_{13}(C)_{42} \\
\left(\gamma^{\mu} C\right)_{13}\left(C \gamma_{\mu}\right)_{42}
\end{array}\right) \quad(\bar{q}-q \rightarrow q-q \text { channel })
$$

Similarly, the matrix $F_{\bar{q} q \rightarrow \bar{q} q}^{S_{3}}$ is selfconverse, but $F_{\bar{q} q \rightarrow q q}^{S_{3}}$ is not. The converse of the latter is

$$
\begin{gathered}
\left(F_{\bar{q} q \rightarrow q q}^{S_{3}}\right)^{-1} \equiv F_{q q \rightarrow \bar{q} q}^{S_{3}}=\left(\begin{array}{cc}
-\frac{1}{2} & \frac{1}{2} \\
-\frac{3}{2} & -\frac{1}{2}
\end{array}\right) \quad\left(\begin{array}{c}
\text { A.16) } \\
\left(\begin{array}{c}
(1)_{12}(1)_{34} \\
\left(\tau_{a}\right)_{12}\left(\tau_{a}\right)_{34}
\end{array}\right)=\underbrace{\left(\begin{array}{cc}
1 / N & 1 / 2 \\
2\left(N^{2}-1\right) / N^{2} & 1 / N
\end{array}\right)}_{F_{\bar{q} q \rightarrow \bar{q} q}^{U N)}}\left(\begin{array}{c}
(1)_{14}(1)_{32} \\
\left(\tau_{a}\right)_{14}\left(\tau_{a}\right)_{32}
\end{array}\right) \quad(\bar{q}-q \rightarrow \bar{q}-q \mathrm{c} \\
1 / 2 \\
=
\end{array}\right. \\
\underbrace{\left(\begin{array}{c}
1 / 2 \\
(N-1) / N \\
-(N+1) / N
\end{array}\right)}_{F_{\bar{q} q \rightarrow q q}^{U(N)}}\left(\begin{array}{l}
\left(\tau_{S}\right)_{13}\left(\tau_{S}\right)_{42} \\
\left(\tau_{A}\right)_{13}\left(\tau_{A}\right)_{42}
\end{array}\right) \quad(\bar{q}-q \rightarrow q-q \text { channel })
\end{gathered}
$$

which will generate the converse of the transformation (A.15).

\section{A.2 Generators of $U(N)$}

We denote the generators of the group $S U(N)$ by $\tau_{a}$ $(a=1, \cdots, N-1)$ and denote $\tau_{0} \equiv \sqrt{2 / N} 1$, where 1 is the $N \times N$ unit matrix, they are normalized by $\operatorname{Tr} \tau_{a} \tau_{b}=2 \delta_{a b}$. The Fierz transformations may be expressed by
In (A.18) $\tau_{S}$ (including $\tau_{0}$ ) and $\tau_{A}$ are respectively symmetric and antisymmetric generators of $U(N)$. It is indicated that the matrix $F_{\bar{q} q \rightarrow \bar{q} q}^{U(N)}$ is selfconverse, but $F_{\bar{q} q \rightarrow q q}^{U(N)}$ is not. The converse of the latter is

$$
\left(F_{\bar{q} q \rightarrow q q}^{U(N)}\right)^{-1} \equiv F_{q q \rightarrow \bar{q} q}^{U(N)}=\left(\begin{array}{cc}
(N+1) / N & 1 / 2 \\
(N-1) / N & -1 / 2
\end{array}\right)
$$

which corresponds to the $U(N)$ Fierz transformation of $q q \rightarrow \bar{q} q$ channel.

It is emphasized that in the discussions of this paper, when $U(N)$ is considered as the flavor-related group $U_{f}\left(N_{f}\right)$, the generators will be denoted by $\left(1_{f}, \tau_{a}\right)$ or $\left(\tau_{S}, \tau_{A}\right)$ and when $U(N)$ is considered as the color-related group $U_{c}\left(N_{c}\right)$, the generators will be denoted by $\left(1_{c}, \lambda_{a}\right)$ or $\left(\lambda_{S}, \lambda_{A}\right)$.

\section{References}

[1] Y. Nambu and G. Jona-Lasinio, Phys. Rev. 122, 345 (1961); 124, 246 (1961).

[2] D.J. Gross and A. Neveu, Phys. Rev. D 10, 3235 (1974).

[3] J. Goldstone, Nuovo Cimento 19, 154 (1961).

[4] D. Weingarten, Phys. Rev. Lett. 51, 1830 (1983); S. Nussinov, Phys. Rev. Lett. 51, 2081 (1983); E. Witten, Phys. Rev. Lett. 51, 2351 (1983); C. Vafa and E. Witten, Nucl. Phys. B234, 173 (1984).

[5] B. R. Zhou, Phys. Lett. B215, 364 (1988); B235, 412 (1990)(E);
Zhou BangRong, Commun. Theor. Phys. 15, 319 (1991); 18, 347 (1992); 19, 377 (1993).

[6] V. A. Miransky, Dynamical symmetry breaking in Quantum Field Theory, World Scientific, Singapore (1993) and the references therein.

[7] D.A. Kirzhnits and A.D. Linde, Phys. Lett. B 42, 471 (1972); S. Weinberg, Phys. Rev. D 7, 2887 (1973); 9, 3357 (1974); L. Dolan and R. Jackew, Phys. Rev. D 9, 3320 (1974 ).

[8] M.A. Sakawa and K. Yazaki, Nucl. Phys. A 504, 668 (1989); S.P. Klevansky, Rev. Mod. Phys. 64, 649 (1992); T. Hatsuda and T. Kunihiro, Phys. Rep. 247, 221 (1994).

[9] D. Ebert, K.G. Klimenko, M.A. Vdovichenko, and A.S. Vshivtsev, Phys. Rev. D 61, 025005 (1999).

[10] B. R. Zhou, Phys. Rev. D57, 3171 (1998); Phys. Lett. B444, 455 (1998); Zhou BangRong, Commun. Theor. Phys. 32, 425 (1999); 33, 451 (2000); 40, 67 (2003); 669 (2003); Wang XiaoMing and Zhou BangRong, ibid. 47, 1081 (2007).

[11] J. C. Collins and M. J. Perry, Phys. Rev. Lett. 34, 1353 (1975); M. Alford, K. Rajagopal, and F. Wilczek, Phys. Lett. B422, 427 (1998); Nucl Phys . B537, 443 (1999); J. Berges and K. Rajagopal, Nucl. Phys. B538, 215 (1999).

[12] R. Rapp, T. Sch ä fer, E. V. Shuryak, and M. Velkovsky, Phys. Rev. Lett. 81, 53 (1998).

[13] M. Buballa, Phys. Rep. 407, 205 (2005).

[14] D. Diakonov, H. Forkel, and M. Lutz, Phys. Lett. B 373, 147 (1996).

[15] B. Vanderheyden and A.D. Jackson, Phys. Rev. D61, 076004 (2000).

[16] S. B. Rüster and D. H. Rischke, Phys. Rev. D69, 045011 (2004). 
[17] Zhou BangRong, Commun. Theor. Phys. 47, 95 (2007); 520 (2007); 695 (2007).

[18] Zhou BangRong, Commun. Theor. Phys. 51, 700 (2009).

[19] W. Pauli, Nuovo Cimento 6, 205 (1957); F. Gürsey, ibid. 7, 411 (1958).

[20] M. Peskin, Nucl. Phys. B175, 197 (1980).

[21] D. Diakonov and V. Petrov, Diquarks in the instanton picture, in 'Quark cluster dynamics', eds. K. Goeke et al., Springer-Verlag, 1993.
[22] A. Smilga and J. J. M. Verbaarschot, Phys. Rev. D51, 829 (1995).

[23] N. D. Mermin and H. Wagner, Phys. Rev. Lett. 17, 1133 (1966); S. Coleman, Commun. Math. Phys. 31, 259 (1973).

[24] Zhou BangRong, Commun. Theor. Phys. 54, 499 (2010)(E). 